\title{
The Relationship Between Bob Burnout and Emotional Labor among College Counselors: Mediating Effect of General Self-Efficacy
}

\author{
Yongmei $\mathrm{Hou}^{1, *}$ and Jiangmin Zheng $^{2}$
}

\author{
${ }^{1}$ Department of Psychology, School of Humanities and Management, Guangdong Medical University, Dongguan, \\ Guangdong, China \\ ${ }^{2}$ School of Biomedical Engineering, Guangdong Medical University, Dongguan, Guangdong, China \\ *Corresponding author. E-mail: 3625321330@qq.com
}

\begin{abstract}
The aim of this study is to explore the characteristics of job burnout, general self-efficacy and emotional labor in college counselors, and analyze the relationship among the 3 variables. Totally 538 college counselors were recruited through Internet from 7 universities in Guangdong Province. They were investigated with Maslach Burnout Inventory-General Survey (MBI-GS), Emotional Labor Scale (ELS) and General Self Efficacy Scale (GSES). The survey results indicate the following four points. First, the scores of MBI-GS, GSES, surface performance, deep performance and natural performance were (2.95 \pm 0.85$),(2.42 \pm .60)$, (4.45 \pm .83$),(4.27 \pm .94)$ and $(3.81 \pm .85)$, respectively. Second, counselors with mild, moderate and severe job burnout accounted for $34.4 \%, 26.8 \%$ and $6.3 \%$, respectively. Third, there was a pairwise correlation among the total score of surface performance, GSES and MBI-GS $(\mathrm{r}=-.104, .247,-.344$, all $P<0.01)$, a pairwise correlation among the total score of deep performance, GSES and MBI-GS $(r=.285,-.235,-.344$, all $P<$ $0.01)$, as well as a pairwise correlation among the total score of natural performance, GSES and MBI-GS $(\mathrm{r}=$ $.316,-.276,-.344$, all $P<0.01$ ). Last, the score Of GSES played a partly mediating effect in the relationship between surface performance and MBI-GS, a partly mediating effect in the relationship between deep performance and MBI-GS, as well as a partly mediating effect in the relationship between natural performance and MBI-GS, with the mediation effect accounting for $14.5 \%, 41.7 \%$ and $39.4 \%$ of the total effect, respectively. It is therefore suggested that emotional labor not only has a direct role on the job burnout in college counselors, but also indirectly affects it through general self-efficacy.
\end{abstract}

Keywords: College Counselors; Job Burnout; General Self-Efficacy; Emotional Labor; Mediating Effect

\section{INTRODUCTION}

Job burnout refers to a long-term response caused by an individual's inability to effectively deal with various continuous pressures at work, which is generally considered to include emotional exhaustion, cynicism and reduced personal accomplishment [1]. Job burnout has a negative impact on individuals' physical and mental health and job performance, as well as the development of organization that individuals belong to [2].

Job burnout can occur in a variety of occupational groups, and practitioners in the helping industry are the high incidence groups. College counselors are not only responsible for the management of students' daily affairs, but also bear the major responsibilities of shaping students' personality and morality, affecting students' academic life, career and psychosocial development [3]. It can be said that college counselors are typical helpers. Previous studies show that counselors are more prone to job burnout than full-time teaching and research personnel in colleges or universities [4], but there are few empirical studies on job burnout of college counselors.

Self-efficacy [5] refers to people's expectation of whether they have the ability to complete a certain task in specific situation, which reflects the subjective evaluation of the extent to which individuals can control the environment and their own behavior. After mastering the corresponding knowledge and skills, self-efficacy becomes the determinant of behavior. It affects people's choice and persistence of activities, and attitude towards difficulties. In the face of difficulties, those with low self-efficacy think more about their own shortcomings, so they bear greater pressure, avoid difficulties and even cancel actions. While those with high self-efficacy tend to choose challenging tasks, establish higher goals, stimulate stronger motivation and will, recover quickly from setbacks, deal with difficulties rationally and achieve goals.

Generally speaking, self-efficacy is a domain specific concept, because individuals may have high self-confidence in one domain, but may not be so in other domains. However, scholars also find a universal sense of self-efficacy, that is, general self-efficacy, which is the 
overall self-confidence that individuals need to face new things or deal with environmental challenges.

To a certain extent, general self-efficacy reflects the habitual self-confidence in coping with new environments or difficulties, and has a significant correlation with specific self-efficacy in various fields, which is convenient for group comparison [4].

Diefendorff et al. (2003) [6] defined emotional labor as a dynamic processing process in which individuals monitor and adjust emotional expression to conform to the expression rules. Diefendorff's cybernetic model takes cybernetic principles as the framework of occurrence mechanism of emotional labor. The core concept of this theory is feedback loop, which includes four main steps (components): input, standard, comparator and output. Input is to input external information into the processing system; Standards represent the goal someone wants to achieve; Comparator compares input with standard. If they are consistent, output (reaction) will be made according to the current state. If they are inconsistent, they will be adjust to make output consistent with standard. Based on this process, Diefendorff puts forward three strategies of emotional labor: surface, deep and natural performance. Because emotional labor needs to suppress or even completely change one's own real emotions to make them consistent with the requirements (of organization or situation), it is easy to make individuals emotional imbalance, and result in fatigue, tension, low satisfaction, and even burnout [7].

Emotional labor, self-efficacy and job burnout are predictors of job performance. Emotional labor is an individual's self-evaluation of the way and effect of emotional management at work, which result is largely affected by self-confidence (self-efficacy); Emotional exhaustion, the core component of job burnout, refers to the phenomenon that someone is unable to effectively manage his own emotions at work, that is, the lack of self-efficacy of emotion management at work; Therefore, there is a logical connection between the three in connotation [8].

On the other hand, empirical studies find that there is a pairwise correlation among emotional labor, general self-efficacy and job burnout [9-13]. Job burnout is a series of explicit behavioral and emotional responses, belonging to outcome variables; Emotional labor is individuals' emotional control and management mode, belonging to deep psychological qualities (distal antecedent variables). Self-efficacy is a psychological quality to overcome difficulties manifested in the choice and reaction of enviroment, belonging to surface psychological qualities (proximal antecedent variables). So, emotional labor should be mediated by general self-efficacy. we can assume general self-efficacy plays an intermediary role between emotional labor and job burnout.

\section{OBJECTS AND TOOLS}

\subsection{Objects}

Through online recruitment, 600 counselors were selected from 7 universities including Shenzhen University, Guangdong Ocean University, South China University of Technology, Southern Medical University, Xinghai Conservatory of Music, Guangzhou Institute of Physical Education and Guangzhou University of Finance and Economics. 538 valid questionnaires were collected, with an effective rate of $89.7 \%$. The age was 24 to 43 years old, with an average of $(32.33 \pm 4.27)$ years old. There were 293 males and 245 females; 353 had a bachelor's degree, 146 had a master's degree and 39 had a doctoral degree.

\subsection{Tools}

\subsubsection{Maslach Burnout Inventory-General Survey, MBI-GS}

It is compiled by Maslach (1997) [2], and revised into Chinese version by Li Chaoping (2003) [14]. There are 16 questions, which are divided into three dimensions: emotional exhaustion, cynicism and reduced personal accomplishment. Likert 7-point scoring method is used to score from 0 to 6 points corresponding to "never" to "very frequent". The higher the total score, the higher the degree of job burnout. In this study, Cronbach's a coefficient of the total scale is 0.894, and Cronbach's a coefficient of three dimensions are $0.843,0.825$ and 0.850 , respectively. $\mathrm{Li}$ Yongxin et al. [15] put forward the critical value of MBI-GS (exhaustion score $\geqslant 25$, cynicism score $\geqslant 11$, reduced personal accomplishment score $\geqslant 16$ ) and divided job burnout into four levels, namely zero burnout (scores on three dimensions of MBI-GS are all lower than the critical values), mild burnout (the score on one dimension is equal to or higher than critical value), moderate burnout (scores on two dimensions are equal to or higher than critical values), and high burnout (scores on three dimensions are equal to or higher than critical values).

\subsubsection{General Self-Efficacy Scale, GSES}

Compiled by Schwarzer (1997) [16] and revised into Chinese version by Wang Caikang et al. (2000) [17]. GSES is a one-dimensional with 10 items. Likert 4-point scoring method is ued to score from 1 to 4 points corresponding to "completely incorrect" to "completely correct". The higher the total score, the higher the general self-efficacy. In this study, Cronbach'a coefficient of GSES is 0.858 . 


\subsubsection{Emotional Labor Scale, ELS}

Compiled by Diefendorff (2005) [18], and revised into Chinese version by Zhong Jian'an (2007) [19]. There are 14 items divided into three dimensions, including surface, deep and natural performance. Likert 5-point scoring method is used to score from 1 to 5 points corresponding to "never" to "always". The higher the total score, the higher the emotional labor. In this study, Cronbach'a coefficients of three subscales are 0.864, 0.830 and 0.815, respectively.

\subsection{Data processing}

SPSS 20.0 is used for statistical analysis. Descriptive statistics is used to calculate the average score and standard deviation of each scale; Pearson product moment correlation is used to explore the correlation between variables; Linear stepwise regression analysis is used to analyze the mediating role of general self-efficacy between emotional labor and job burnout.

\section{RESULTS AND ANALYSIS}

\subsection{Status of job burnout of college counselors}

The incidence of job burnout in this group is $67.5 \%$ $(363 / 583)$, and the detection rates of mild, moderate and serious job burnout are $34.4 \%, 26.8 \%$ and $6.3 \%$, respectively.

\subsection{Descriptive statistics and correlation analysis of each variable}

It can be seen from table 1 that there is a pairwise correlation among the total score of surface performance, GSES and MBI-GS ( $\mathrm{r}=-.104, .247,-.344$, all $\mathrm{P}<0.01)$; a pairwise correlation among the total score of deep performance, GSES and MBI-GS $(r=.285,-.235,-.344$, all $\mathrm{P}<0.01)$; a pairwise correlation among the total score of natural performance, GSES and MBI-GS ( $\mathrm{r}=.316$, $276,-.344$, all $\mathrm{P}<0.01)$.

Table 1. Correlation analysis of scores of various scales and dimensions

\begin{tabular}{|c|c|c|c|c|c|c|c|c|c|c|}
\hline & \multirow{2}{*}{$\begin{array}{c}\mathrm{M} \\
4.452\end{array}$} & $\mathrm{SD}$ & 1 & 2 & 3 & 4 & 5 & 6 & 7 & \multirow[t]{2}{*}{8} \\
\hline 1.Surface performance & & .831 & & & & & & & & \\
\hline 2.Deep performance & $\begin{array}{l}4.273 \\
3.809\end{array}$ & $\begin{array}{r}.938 \\
850\end{array}$ & -.013. & $153^{* * *}$ & & & & & & \\
\hline $\begin{array}{l}\text { 3. Natural performance } \\
\text { 4.Total score of GSES }\end{array}$ & $\begin{array}{l}3.809 \\
2.422\end{array}$ & $\begin{array}{l}.850 \\
.602\end{array}$ & $\begin{array}{l}-.027 \\
-.104^{*}\end{array}$ & $\begin{array}{l}.153 \\
.285^{* *}\end{array}$ & $.316^{* *}$ & & & & & \\
\hline 5. Emotional exhaustion & 3.220 & .963 & $.271^{* *}$ & $-.331^{* *}$ & $-.294^{* *}$ & $-.267^{* *}$ & & & & \\
\hline 6. Cynicism & 2.144 & .980 & $.200^{* *}$ & $-.130^{* *}$ & $-.178^{* *}$ & $-.389^{* *}$ & $.631^{* *}$ & & & \\
\hline 7. Low sense of achievement & 3.062 & .871 & $.113^{* *}$ & $-.264^{* *}$ & $-.341^{* *}$ & $-.756^{* *}$ & $.519^{* *}$ & $.134^{* *}$ & & \\
\hline 8.Total score of MBI-GS & 2.951 & .852 & $.247^{* *}$ & $-.235^{* *}$ & $-.276^{* *}$ & $-.344^{* *}$ & $.884^{* *}$ & $.870^{\text {*** }}$ & $.930^{* *}$ & \\
\hline
\end{tabular}

Note: $* p<0.05,{ }^{* *} p<0.01$ (The same below)

\subsection{Mediating effect test of general self-efficacy on emotional labor and job burnout}

According to the mediation effect test method proposed by Wen Zhonglin et al. [20], the score of each dimension of

ELS is used as independent variable respectively, MBI-GS total score is used as dependent variable, and GSES total score is used as intermediary variable. The results are shown in Table 2, Table 3 and Table 4, respectively.

\subsubsection{Mediating effect of general self-efficacy between surface performance and job burnout}

Table 1. Mediating effect of general self-efficacy between surface performance and job burnout

\begin{tabular}{clcccc}
\hline Step & Dependent variable & Independent variable & $\beta$ & $t$ & $R^{2}$ \\
\hline Step 1(c) & MBI-GS total score & Surface performance & 0.247 & $6.305^{* *}$ & 0.061 \\
Step 2(a) & GSES total score & Surface performance & -0.104 & $-2.934^{*}$ & 0.009 \\
Step 3(c') & MBI-GS total score & Surface performance & 0.195 & $3.836^{* *}$ & 0.123 \\
(b) & & GSES total score & -0.344 & $-8.146^{* *}$ &
\end{tabular}

It can be seen from Table 2 that in the first step, surface performance positively predicts MBI-GS total score, with an significant regression coefficient of (0.247); In the second step, surface performance negatively predicts GSES total score, with an significant regression coefficient of (-0.104); In the third step, after controlling the influence of GSES on MBI-GS total score, surface performance can still positively predict MBI-GS total score, with an significant regression coefficient of (0.195), that is, after adding the intermediary variable GSES total score between dependent variable MBI-GS total score and independent variable surface performance, the absolute value of regression coefficient between MBI-GS total score and surface performance decreases. It can be seen that GSES 
has a significant partial mediating effect on the relationship between surface performance and MBI-GS total score, with an mediating effect accounting for $14.5 \%$ of the total effect (i.e. effect $\mathrm{M}=\mathrm{ab} / \mathrm{c}=(-0.104) \times(-0.344) / 0.247 \times$ $100 \%=14.5 \%$ ).

Table 2. Mediating effect of general self-efficacy between deep performance and job burnout

\begin{tabular}{rlrrrr}
\hline Step & Dependent variable & Independent variable & $\beta$ & $t$ & $R^{2}$ \\
\hline Step 1(c) & MBI-GS total score & Deep performance & -0.235 & $-5.984^{* *}$ & 0.055 \\
Step 2(a) & GSES total score & Deep performance & 0.285 & $7.134^{* *}$ & 0.081 \\
Step 3(c') & MBI-GS total score & Deep performance & -0.147 & $-4.033^{* *}$ & 0.183 \\
(b) & & GSES total score & -0.344 & $-8.146^{* *}$ &
\end{tabular}

It can be seen from Table 3 that in the first step, deep performance negatively predicts the total score of MBI-GS, with an significant regression coefficient of $(-0.235)$; In the second step, deep performance positively predicts the total score of GSES, with an significant regression coefficient of (0.285); In the third step, after controlling the influence of GSES on MBI-GS total score, deep performance can still negatively predict MBI-GS total score, with an significant regression coefficient of $(-0.147)$, that is, after adding the intermediary variable GSES total score between dependent variable MBI-GS total score and independent variable deep performance, the

\subsubsection{Mediating effect of general self-efficacy between deep performance and job burnout}

Table 3. Mediating effect of general self-efficacy between natural performance and job burnout

\begin{tabular}{clcccc}
\hline Step & Dependent variable & Independent variable & $\beta$ & $t$ & $R^{2}$ \\
\hline Step 1(c) & MBI-GS total score & Natural performance & -0.276 & $-7.418^{* *}$ & 0.217 \\
Step 2(a) & GSES total score & Natural performance & 0.316 & $7.134^{*}$ & 0.109 \\
Step 3(c') & MBI-GS total score & Natural performance & -0.239 & $-5.667^{* *}$ & 0.183 \\
(b) & & GSES total score & -0.344 & $-8.146^{* *}$ & \\
\hline
\end{tabular}

It can be seen from Table 4 that in the first step, natural performance negatively predicts MBI-GS total score, with an significant regression coefficient of $(-0.276)$; In the second step, natural performance positively predicts GSES total score, with an significant regression coefficient of (0.316); In the third step, after controlling the influence of GSES on MBI-GS total score, natural performance can still negatively predict MBI-GS, with an significant regression coefficient of (-0.239), that is, after adding the intermediary variable GSES total score between the dependent variable MBI-GS total score and independent variable natural performance, the absolute value of the regression coefficient between natural performance and MBI-GS total score decreases. It can be seen that GSES total score has a significant partial mediating effect on the relationship between natural performance and MBI-GS total score, with an mediating effect accounting for $39.4 \%$ of the total effect (i.e. effect $\mathrm{M}=\mathrm{ab} / \mathrm{c}=(0.316) \times$ $(-0.344) /(-0.276) \times 100 \%=39.4 \%)$.

\section{DISCUSSION}

The incidence rates of mild, moderate and serious job burnout in this group are $34.4 \%, 26.8 \%$ and $6.3 \%$, respectively, and counselors in our study have a low level of general self-efficacy [16-17] and a high level of emotional labor [18-19], which is consistent with the results of previous studies [21-23], suggesting that college counselors generally have obvious job burnout, high emotional labor and low general self-efficacy.

There are direct and indirect effects between college counselors' emotional labor and job burnout.

On one hand, There is a significant correlation between each dimension of ELS and MBI-GS total score, indicating that there is a direct effect between emotional labor and job burnout, which is consistent with the research results of Zhao Xiaobin et al. [21]. In other words, the higher the level of emotional labor, the more serious the job burnout. On the other hand, there is an indirect effect between emotional labor and job burnout. It is manifested in the following ways: emotional labor - general self-efficacy job burnout, that is, general self-efficacy plays a partial 
intermediary role between emotional labor and job burnout. People with higher emotional labor often have lower general self-efficacy. They lack self-confidence to control the environment and self-behavior, think themselves are incompetent, and often feel nervous, frustrated and afraid at work, resulting in job burnout.

\section{CONCLUSION}

This study initially reveals the relationship between emotional labor and job burnout of college counselors, and verifies the following hypothesis: General self-efficacy plays a partial intermediary role between emotional labor and job burnout.

To sum up, both emotional overwork and low general self-efficacy are important reasons for college counselors' job burnout. In other words, personality traits and cognitive style are two basically influencing factors of college counselors' job burnout. According to this conclusions, the following recommendations are made: The intervention of job burnout of college counselors should start with strengthening the professional construction of counselors, improving their professional core competence, work efficiency and self-efficacy; At the same time, we should strengthen mental health education and psychological quality training to help them scientifically regulate emotional activities at work, appropriately reduce emotional labor intensity and avoid excessive emotional labor. In the future, we can add longitudinal research data to further verify the relationship between college counselors' emotional labor and job burnout.

\section{ACKNOWLEDGMENT}

This study is sponsored by 2020 research project of the 13th five-year plan of Educational Science in Guangdong Province (Project No. 2020GXJK386)

\section{REFERENCES}

[1] Maslach C, Schaufeli WB, Leiter MP. Job burnout[J]. Annual Review of Psychology, 2001, 52: 397-422.

[2] Maslach C, Leiter MP. The truth about burnout: How organizations cause personal stress and what to do about it?[M]. San Francisco: Jossey-Bass Inc., 1997.

[3] Ministry of Education of the People's Republic of China. Opinions on strengthening the construction of counselors and head teachers in colleges and universities [EB/OL].

http//old.moe.gov.cn//publicfiles/business/htmlfiles/moe /moe_512/201006/88984.html.
[4] Meng Yong. Psychological stress and job burnout of college counselors [J]. Journal of Clinical Physical and Mental Diseases. 2008, 14(1): 69-71.

[5] Bandura A. Self-efficacy: Toward a unifying theory of behavioral change [J]. Psychological Review, 1977, 84: 191-215.

[6] Diefendorff JM, GosserandRH. Understanding the emotional labor process: A control theory perspective [J]. Journal of Organizational Behavior, 2003, 24(4): 945-959.

[7] J. Andrew Morris, Daniel C. Feldman. The dimensions, antecedents, and consequences of emotional labor[J]. The Academy of Management Review, 1996, 21(4): 986- 1010.

[8] Xu Weimin, Song Tizhong. The impact of emotional labor on employees' job performance [J]. Business Research, 2013, 4(1): 97-101.

[9] Li Yongxin. Relationship between emotional labor, job burnout and job satisfaction of medical staff [J]. Chinese Journal of Nursing, 2018, 44 (6): 506-509.

[10] Yin HB, Huang SH, Chen GW. The relationships between teachers' emotional labor and their burnout and satisfaction: A meta-analytic review[J]. Educational Research Review, 2019, 28:1-18.

[11] Fernando DB. Stressors, self-efficacy, coping resources, and burnout among primary and secondary school teachers in Spain [J]. Educational Psychology, 2006, 26(4): 519- 539.

[12] Friedman IA, Farber BA. Professional self-concept as a predictor of teacher burnout [J]. Journal of Educational Research, 1992, 86(1): 28-35.

[13] Lin Jiaojiao. Research on the relationship between preschool teachers' emotional labor and self-efficacy [D]. East China Normal University, 2016, 06.

[14] Li Chaoping, Shi Kan. The impact of distributive justice and procedural justice on job burnout [J]. Journal of Psychology, 2003, 35(5): 677-684.

[15] Li Yongxin, Li Yimin. Preliminary discussion on the evaluation criteria of job burnout [J]. Psychological Science, 2006, 29(1): 148- 150.

[16] Schwarzer R, Aristi B. Optimistic self-beliefs: Assessment of general perceived self-efficacy in thirteen cultures [J]. Word Psychology, 1997, 3(1-2): 177-190. 
[17] Wang Caikang, Liu Yong. Correlation between general self-efficacy and trait anxiety, state anxiety and test anxiety [J]. Chinese Journal of Clinical Psychology, 2000, 8(3): 56-57.

[18] Diefendorff JM, Gosserand RH. The dimensionality and antecedents of emotional labor strategies [J]. Journal of Vocational Behavior, 2005, 66(3): 339-357.

[19] Zhong Jian'an, Lin Jian, Zhang Yuanyuan. Study on the relationship between emotional expression rules, regulation strategies and job burnout $[\mathrm{J}]$. Chinese Journal of Applied Psychology, 2007, 13(2): 144 - 148.

[20] Wen Zhonglin, Hou Jietai, Zhang Lei. Comparison and application of regulatory effect and mediating effect [J]. Acta Psychologica Sinica, 2005, 37(2): 268 - 274.
[21] Zhao Xiaobin, Meng Hua. Emotional labor, organization fairness and job burnout of counselors in independent colleges [J]. Secretary, 2018, 36(6): 48-57.

[22] Zhao Yan. Research on the relationship between college counselors' professional identity, professional self-concept and job burnout [D]. Harbin: Harbin Normal University, 2013, 05.

[23] Qi Xinyi. Research on overcoming job burnout of college counselors -- taking some colleges and universities in Shanghai as examples [D]. East China Normal University, 2016, 05. 